\title{
Peak load minimization of an e-bus depot: impacts of user-set conditions in optimization algorithms
}

\author{
Enrico Toniato ${ }^{1}$, Prakhar Mehta ${ }^{2 *}$, Stevan Marinkovic ${ }^{3}$ and Verena Tiefenbeck ${ }^{2,4}$ \\ From The 10th DACH+ Conference on Energy Informatics \\ Virtual. 13-17 September 2021
}

\author{
*Correspondence: \\ prakhar.mehta@fau.de \\ ${ }^{2}$ School of Business, Economics and \\ Society, FAU Erlangen-Nuremberg, \\ Lange Gasse 20, 90403 Nuremberg, \\ Germany \\ Full list of author information is \\ available at the end of the article
}

\begin{abstract}
The transport sector is responsible for $25 \%$ of global $\mathrm{CO}_{2}$ emissions. To reduce emissions in the EU, a shift from the currently 745,000 operating public buses to electric buses (EBs) is expected in the coming years. Large-scale deployments of EBs and the electrification of bus depots will have a considerable impact on the local electric grid, potentially creating network congestion problems and spikes in the local energy load. In this work, we implement an exact, offline, modular multi-variable mixed-integer linear optimization algorithm to minimize the daily power load profile peak and optimally plan an electric bus depot. The algorithm accepts a bus depot schedule as input, and depending on the user input on optimization conditions, accounts for varying time granularity, preemption of the charging phase, vehicle-to-grid (V2G) charging capabilities and varying fleet size. The primary objective of this work is the analysis of the impact of each of these input conditions on the resulting minimized peak load. The results show that our optimization algorithm can reduce peak load by $83 \%$ on average. Time granularity and V2G have the greatest impact on peak reduction, whereas preemption and fleet splitting have the greatest impact on the computational time but an insignificant impact on peak reduction. The results bear relevance for mobility planners to account for innovative fleet management options. Depot infrastructure costs can be minimized by optimally sizing the infrastructure needs, by relying on split-fleet management or V2G options.
\end{abstract}

Keywords: Multi-variable optimization, Preemption, Vehicle-to-grid (V2G)

\section{Introduction}

The transport sector accounts for $25 \%$ of the global CO2 emissions (IEA 2020). Electric vehicles (EVs), ideally powered by renewable energy sources, have the potential to drastically cut these emissions. Public transport fleets, of which buses are the most widely used form in the EU (2050 long-term strategy - Climate Action), offer a massive electrification opportunity. Currently, about 745,000 buses operate in the EU, accounting for $55.7 \%$ of all public transport journeys and ferrying up to 32.1 billion passengers per year (2050 
long-term strategy - Climate Action). As of 2019, only 4,500 electric buses operated in the EU, with a doubling in new registrations from 2018 to 2019 (Global EV outlook). Encouragingly, the use of electric buses (EBs) is expected to increase considerably in the coming years, spurred by greener policies and government incentives (Buses - ACEA European Automobile Manufacturers' Association).

The introduction of electric buses in the public transport fleet will help governments fulfil requirements of recent environmental laws, meant to make the EU climate-neutral by 2050 (Rahman and Shrestha 1993), but at the same time poses several challenges. Large-scale deployments of EBs and EVs and the linked recharging infrastructures can have a significant impact on the local electric grid, creating network congestion issues and spikes in the electricity load profile (Clairand et al. 2020; Clement-Nyns et al. 2010; Valckx et al. 2019; Das et al. 2020; Ramabhotla et al. 2016), especially under uncontrolled charging activities. To mitigate such problems, many techniques have been suggested in literature. These include direct and indirect peak minimization through various optimization techniques - linear, mixed-integer linear, quadratic or dynamic programming - under a variety of optimization conditions such as different bus fleet sizes, battery sizes, preemption of the charging phase (that is, discrete charging slots with charging tasks unrelated to past or future charging tasks) and vehicle-to-grid (V2G), among others (see Related work section). Most existing approaches, however, consider a fixed set of optimization conditions (e.g., a linear optimization approach to minimize costs with fixed time intervals without V2G) and report optimal energy peaks without analyzing the impact of user-set optimization conditions on the results.

This leads us to the following two research questions:

RQ1: What is the impact of different user-set conditions during optimization (time granularity of the optimization, preemption, V2G and fleet size) on the final objective of peak minimization?

RQ2: What is the corresponding impact on computational time?

We answer these questions by implementing an exact, offline, modular multi-variable mixed-integer linear optimization algorithm to minimize the daily power load profile peak and optimally plan an electric bus depot, under different user-set conditions. The algorithm was developed as part of Hitachi ABB Power Grids' (ABB 2020) projects for EB depot planning, hence with a focus on peak minimization without the inclusion of operating costs. Key results indicate that our optimization algorithm can reduce peak power load by $83 \%$ on average. Further, the results highlight the importance of enabling or disabling different user-set conditions in the optimization, which have impacts on both the reduction of the peak and the computational time. A larger time granularity of the optimization (i.e., smaller time interval) and enabling V2G have the greatest impact on peak reduction, whereas enabling preemption and fleet splitting have the greatest impact on the computational time reduction but an insignificant impact on peak reduction. These results imply lower initial investment costs due to optimum equipment sizing. EB depot planners can account for the impacts of these user-set conditions, such as the availability of V2G infrastructure and fleet-splitting, in order to manage EB fleet operation effectively.

\section{Related work}

Coordinated control strategies to flatten the EV charging load are divided into two types. Direct load flattening algorithms (Nguyen et al. 2014; Jahic et al. 2019; Turker and Colak 
2018; Erdogan et al. 2018; Ranjan et al. 2014; Arango Castellanos et al. 2019) flatten the load profile by coordinating vehicle charging, shifting loads to minimize the peak. By contrast, the objective of indirect load flattening algorithms (Houbbadi et al. 2019; Tang and Zhang 2017; Xu and Wong 2011; Rotering and Ilic 2011; Sundström and Binding 2010 ) is to minimize the cost of charging. The former is a more relevant scenario when considering EB depots, since it is common for transport companies to buy electricity at a contracted rate from the local utility (Jahic et al. 2019). In such cases, the objective function of the type $\min \left(\max \left(P_{t}\right)\right)$ (Nguyen et al. 2014), where $P_{t}$ is the power consumed at an instant $t$, is usually translated into the quadratic form $\min \left(P_{t}\right)^{2}$ (Houbbadi et al. 2019) that minimizes the load variance and spikes. Along with the objective function, several linear constraints must be taken into account, such as the arrival and departure of vehicles, the maximum charging power and the current state of charge (SOC).

Several methods to solve the stated optimization model can be deployed. These methods include linear programming (LP) (Hu et al. 2011; Sundström and Binding 2010; Turker and Colak 2018; Nageshrao et al. 2017) and mixed-integer linear programming (MILP) (Franco et al. 2015; Clemente et al. 2014; Ranjan et al. 2014), which are usually the fastest ones in terms of computational time. Since linear optimization cannot be directly exploited for peak minimization with a quadratic objective function, a workaround is presented in (Nguyen et al. 2014) through a bisection approach, which is adopted in this work and explained in the Methodology section. Other studies propose a quadratic programming approach (QP) (Houbbadi et al. 2019; Turker and Colak 2018) and dynamic programming (DP) (Xu and Wong 2011; Rotering and Ilic 2011; Korkas et al. 2017; Škugor and Deur 2014), and it has been shown that the computational time of DP is longer compared to QP with a negligible difference in the resulting charging profiles (Valckx et al. 2019).

In addition to the methods stated above, the existing literature proposes various heuristics (rule of thumb approaches), greedy algorithms, stochastic programming and even particle swarm and genetic algorithms (Jahic et al. 2019; Gao et al. 2018; Arango Castellanos et al. 2019; Yang et al. 2019). Many of these approaches have either longer computation times (particle swarm and genetic algorithms) or result in sub-optimal solutions and are applicable in specific scenarios only (heuristics) (Nageshrao et al. 2017).

Approaches are also classified and differentiated into open-loop (offline) and closedloop (online/real-time) based on the availability of external information about the vehicles and its parameters. An open-loop approach can be used if all information is available and sufficiently accurate (day-ahead scheduling) (Nguyen et al. 2014; Hu et al. 2011; Houbbadi et al. 2019; Xu and Wong 2011; Rotering and Ilic 2011). These methods can be computationally slower than closed-loop approaches since there is no need for immediate control action. If this information is not available or difficult to predict, a real-time approach can utilize closed-loop obtained information to solve the problem (Tang and Zhang 2017; Koutsopoulos and Tassiulas 2012; Xie et al. 2016; Yang et al. 2019; Erdogan et al. 2018). In this case, most common methods use Dynamic Programming and Model Predictive Control (MPC) to find a solution to the optimization task.

With further consideration of the nature of parameters and variables involved in the computation, peak shaving strategies can be divided even further. First, some studies speed up computation by considering an aggregate battery model of all batteries, instead of tracking the SOC of every single vehicle with an independent variable. Second, by 
employing a continuously controllable charging power, it is possible to solve the problem with classic optimization methods such as LP and QP. However, if the charging power is assumed to be fixed, the decision space is discrete and thus integer programming techniques must be deployed. Third, most studies consider the scheduling job to be preemptable, which implies that the controller can arbitrarily start or interrupt the charging of the connected vehicles at any time (Sundström and Binding 2010; Franco et al. 2015). This choice results in a faster calculation of the optimal solution for the corresponding linear programming model and a generally lower peak (Erdogan et al. 2018), although it can lead to battery degradation and should be avoided (Janovec and Koháni 2019). For this reason, some papers consider the charging procedure to be non-preemptable (Nageshrao et al. 2017; Koutsopoulos and Tassiulas 2012; Clemente et al. 2014).

Table 1 organizes relevant literature reporting the values of nine different parameters relevant to the electric vehicle charging optimization problem. Since some studies do not explicitly report the values of the parameters, they are deduced by the authors based on critical analyses of the concerned studies. As can be seen from the table, studies on electric buses as well as electric vehicles, plug-in electric vehicles and generic power loads were accounted for. While there are obvious differences between these application contexts, insights are shared across the cited literature to improve the optimization result. For instance, an EB's schedule is usually known in advance but EV arrival and departure time at parking lots are often unknown. Nevertheless, the algorithms used for optimizing the charging process of the vehicles are, in many cases, interchangeable. Table 1 does not include computational time, since most cited literature does not state it. This further highlights the contribution of this work as computational times based on user-set optimization conditions are also presented.

The algorithm developed in this work is part of a planning tool for the construction of an electric bus depot with minimal infrastructure sizing. It is modular thanks to the ability to add and remove optimization constraints "on-demand" without the need to update the deployment's source code. This allows the authors to analyze the impact of each optimization variable and parameter on the peak load minimization and the computational time. To the knowledge of the authors, this is an aspect previously not documented in existing literature.

\section{Methodology}

Optimization model

The objective of this research is to minimize the maximum peak of the daily power load profile absorbed from the grid, $P_{G_{t}}$ :

$$
\min \max _{t} P_{G_{t}}
$$

It is possible to formulate the problem using a set of binary decision variables that establish the charging or discharging command for each vehicle of the fleet, for each time interval considered during the day. We operate on two different sets, the vehicles $i \in[1, N]$ to be recharged and the discrete time slots $t \in[0, T]$. The quadratic objective function therefore becomes:

$$
\min \sum_{t=0}^{T} P_{G_{t}}^{2}
$$




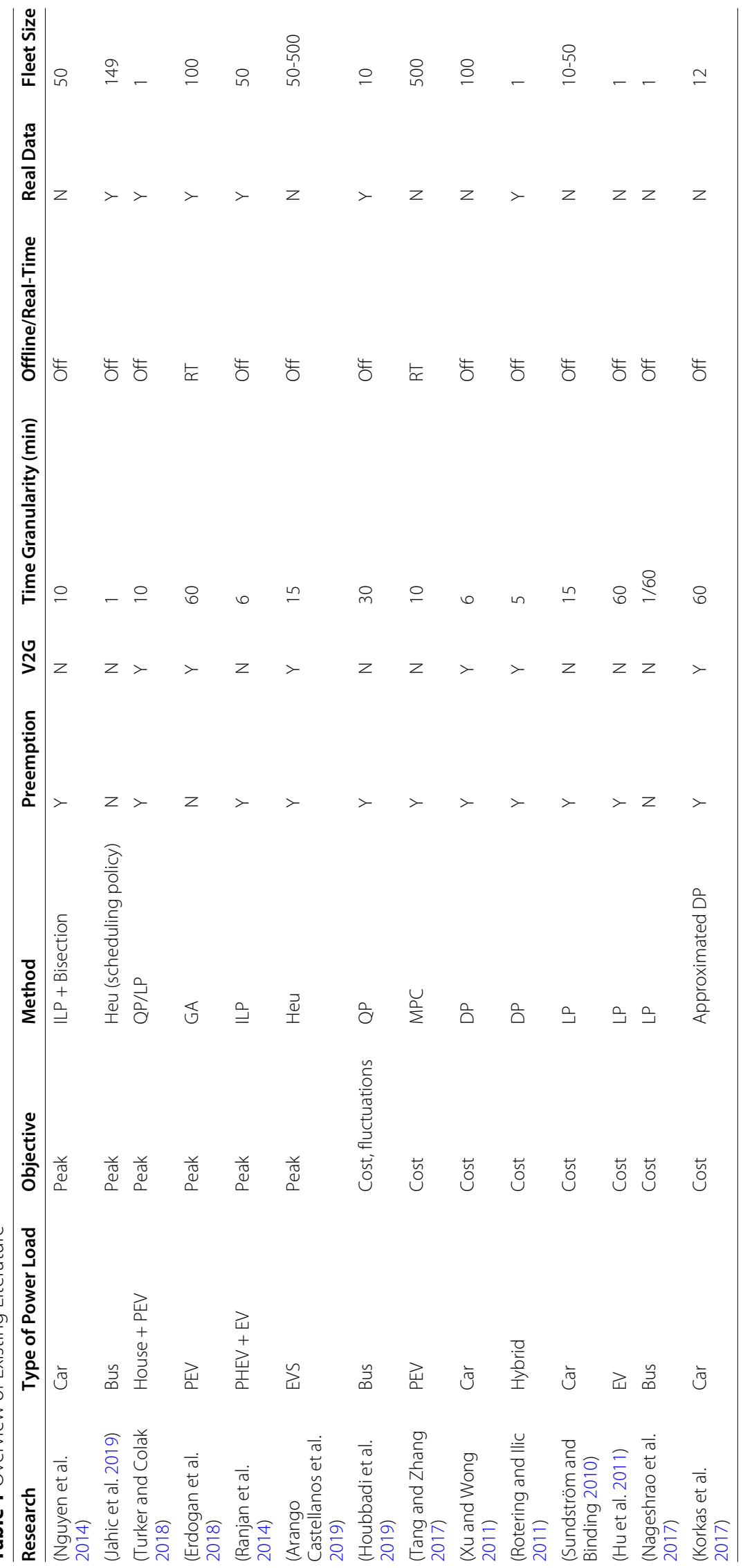




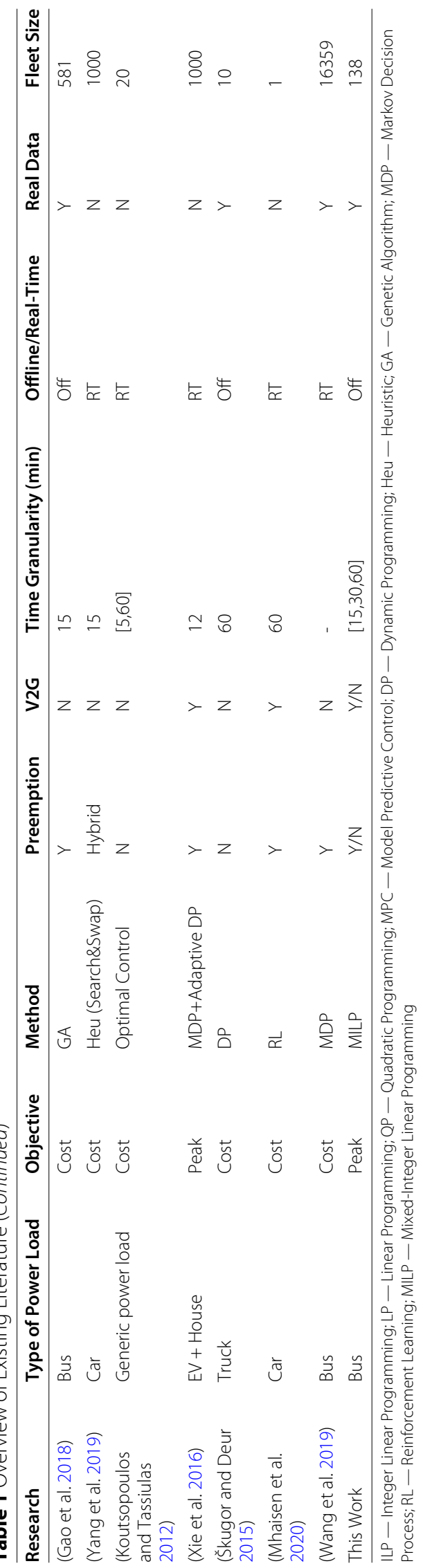


Table 2 Parameters

\begin{tabular}{llll}
\hline Symbol & Range & Unit & Description \\
\hline atDepot $i, t$ & $0 / 1$ & - & $\begin{array}{l}\text { True if bus } i \text { is at depot at time } t . \\
\text { Calculated using the arrival time and departure time of the bus from the } \\
\text { depot. Based on the time granularity of the optimization, the arrival time } \\
\text { is rounded up and the departure time is rounded down. } \\
\text { The state of charge of the battery at arrival, known for each bus from its } \\
\text { schedule. }\end{array}$ \\
SOC $_{\text {Arr, } i}$ & {$[5,100]$} & $\%$ & $\begin{array}{l}\text { The desired SOC at departure. } \\
\text { SOC }\end{array}$ \\
$\Delta$ Dep,$i_{i}$ & 100 & $\%$ & $\begin{array}{l}\text { Percentage change in SOC of each bus i using the fixed charging rate in } \\
\text { kW and given battery capacity }(\mathrm{kWh}) \text { in a single time interval } t .\end{array}$ \\
\hline
\end{tabular}

where

$$
P_{G_{t}}=\eta_{b} * \eta_{c h g} * P_{c h g} * \sum_{i=1}^{N}\left(x_{i, t}-y_{i, t}\right)
$$

$P_{c h g}$ stands for the power capacity of the charger, $\eta_{c h g}$ represents the charger efficiency, $\eta_{b}$ represents the electric bus battery's charge-discharge efficiency and $x_{i, t}$ and $y_{i, t}$ are binary optimization variables which have a value of 1 and 0 when the vehicle is charging, and vice-versa when the vehicle is discharging. Using the approach described in (Hu et al. 2011), the quadratic formulation is translated into a mixed-integer linear programming formulation with an objective function as shown in Eq. 4, and an additional constraint in Eq. 5 reduced iteratively. Optimization parameters and further constraints of the optimization problem are outlined in Tables 2 and 3, respectively, and explained further in the sub-section Modular nature of the optimization.

$$
\begin{aligned}
& \min \sum_{t} P_{G_{t}} \\
& \text { s.t. } P_{G_{t}} \leq \text { upperBound } \forall t
\end{aligned}
$$

The intuition is that there is only one optimal minimum value $b$ for $P_{G_{t}}$ since the charging power is considered constant. This value has a lower bound $b_{m}=0$ (no vehicles charged) and an initial upper bound $b_{M}=P_{c h g} \times N$ where $\mathrm{N}$ is the number of vehicles in the fleet. The value of $b$ is iteratively recomputed as

$$
b=\frac{b_{m}+b_{M}}{2}
$$

$\left[b_{m}, b_{M}\right]$ is replaced with $\left[b, b_{M}\right]$ if the integer linear programming problem is not feasible or with $\left[b_{m}, b\right]$ in the alternate case. The optimal solution is found when the boundaries converge. Figure 1 displays the algorithm in a flow chart.

\section{Modular nature of the optimization}

A key speciality of the optimization algorithm developed in this work is its modular nature. Additional constraints are included in the optimization model only if particular user-set conditions (see sub-section Scenarios) are toggled on/off to evaluate their impact. The need for additional constraints arises if V2G must be enabled or preemption disabled, but does not arise in case of varying the length of the discrete time slots or splitting the fleet into sub-fleets. Table 3 explains how this is employed. $x_{i, t}$ represent the binary variables to indicate if each bus $i$ is charging in every time-slot $t$. In the case of enabling V2G charging capabilities, a new set of binary variables $y_{i, t}$ are created, while the $z_{i, t}$ variables are only activated when preemption is disabled. 
Table 3 Constraints

\begin{tabular}{|c|c|}
\hline Constraint & Description \\
\hline$x_{i, t} \leq \operatorname{atDepot}_{i, t} \forall i, t$ & Charge only if vehicle at depot \\
\hline$y_{i, t} \leq$ atDepot $_{i, t} \forall i, t$ & Discharge to grid only if vehicle at depot (V2G) \\
\hline$x_{i, t}+y_{i, t} \leq 1 \forall i, t$ & Discharge to grid only if vehicle not charging (V2G) \\
\hline $\operatorname{soc}_{i, t=a \text { arrival }}=S O C_{A r r, i} \forall i$ & $\begin{array}{l}\text { SOC on arrival constrained to be equal to the calculated parameter, known from } \\
\text { calculation based on the known schedule }\end{array}$ \\
\hline $\begin{array}{ll}\text { SOC }_{i, t=\text { departure }} & \geq \\
\text { SOC }_{\text {Dep }, i} \forall i & \end{array}$ & $\begin{array}{l}\text { The SOC of a bus at departure must be equal to a predefined level } S O C_{D e p, i} \text {, set } \\
\text { to } 100 \%\end{array}$ \\
\hline $\begin{array}{l}\operatorname{SOC}_{i, t}=\operatorname{SOC}_{i, t-1}+\Delta \mathrm{SOC}_{i} \times \\
\left(x_{i, t-1}-y_{i, t-1}\right) \forall i, t\end{array}$ & $\begin{array}{l}\text { SOC Balance: The SOC at time } t \text { for bus } i \text { is equal to the SOC in the previous time } \\
\text { slot plus/minus the amount charged/discharged in the current time slot. }\end{array}$ \\
\hline$z_{i, t} \leq x_{i, t} \forall i, t$ & Constraints employed for 'No-Preemption Condition' \\
\hline \multicolumn{2}{|l|}{$z_{i, t} \leq x_{i, t-1} \forall i, t$} \\
\hline$z_{i, t} \geq x_{i, t-1}+x_{i, t}-1 \forall i, t$ & $\begin{array}{l}\text { By default, } x_{i, t} \text { variables are independent of each other with respect to the time } \\
\text { slot. This allows the bus recharging process to be interrupted and restarted later } \\
\text { (preemption). }\end{array}$ \\
\hline$\sum_{t} x_{i, t}-\sum_{t} z_{i, t} \leq 1 \forall i, t$ & $\begin{array}{l}\text { If there is a requirement to prevent the interruption of the charging phase, the } \\
\text { following constraints are introduced. The first } 3 \text { equations derive from a common } \\
\text { trick used in optimization to assign the value } 1 \text { to a variable ( } z_{i, t} \text { in this case) if and } \\
\text { only if both other variables are equal to } 1 \text { (in this case the charging command in } \\
\text { the time slot } t \text { and }(t-1) \text { ). The last equation constrains the number of occasions } \\
\text { in which the vehicle is being charged at } t \text { but not in }(t-1) \text { to be } 1 \text {. Effectively, } \\
\text { this leads to the optimization charging a bus in a single charging process across } \\
\text { multiple time slots until the battery is fully charged. }\end{array}$ \\
\hline
\end{tabular}

\section{Case study}

The data used to evaluate the optimization model comprises the daily schedule (arrival and departure times at the bus depot) of 138 diesel buses over a 24-hour period, obtained from Hitachi ABB Power Grids' client (ABB 2020) and can be found in the linked https:// github.com/tonxxd/depot_optimization. There are three types of buses, as shown in Table 4. Since the data set is based on conventional fossil-fuel vehicles, the distances travelled in a day are too large for an electric bus to traverse with one full charge. Hence, the distance travelled during service is shortened by a factor of 1.6 in order to not lose trip data, while keeping the departure and arrival times of the buses the same. The number of buses parked at the depot during the day is not constant: most of the buses are parked in the depot during the night and leave in the morning for service, as can be seen in Fig. 2.

The DC charging power $P_{\text {chg }}$ was set at $150 \mathrm{~kW}$ based on industry standards (Hitachi ABB Power Grids). Each bus is charged to $100 \%$ SOC at departure, and allowed a depth of discharge of $95 \%$. In order to simulate an electric bus depot with the same schedule, these buses are assumed to be electric with a 95\% charge-discharge efficiency $\eta_{b}$ and the SOC for each bus $i$ on arrival at the bus depot is calculated based on the energy consumption $E_{b}$ as described in Table 4, the bus battery capacity $E_{b a t}$ and distance travelled (dist $\left.{ }_{i}\right)$ using the following equation:

$$
S O C_{A r r, i}=\left(1-\frac{\operatorname{dist}_{i} * E_{\text {bus }_{i}}}{\eta_{b} * E_{b a t_{i}}}\right) * 100 \%
$$

Each optimization run considers a random set of 100 buses from the original dataset, and 50 runs are employed in each scenario in order to achieve a meaningful distribution of results. The computation of the optimal schedule (find the $x_{i, t}, y_{i, t}$ that minimizes $\max P_{G_{t}}$ ) is achieved using the commercial solver Gurobi while the input optimization problem is formulated in Python using the Python-MIP library. 


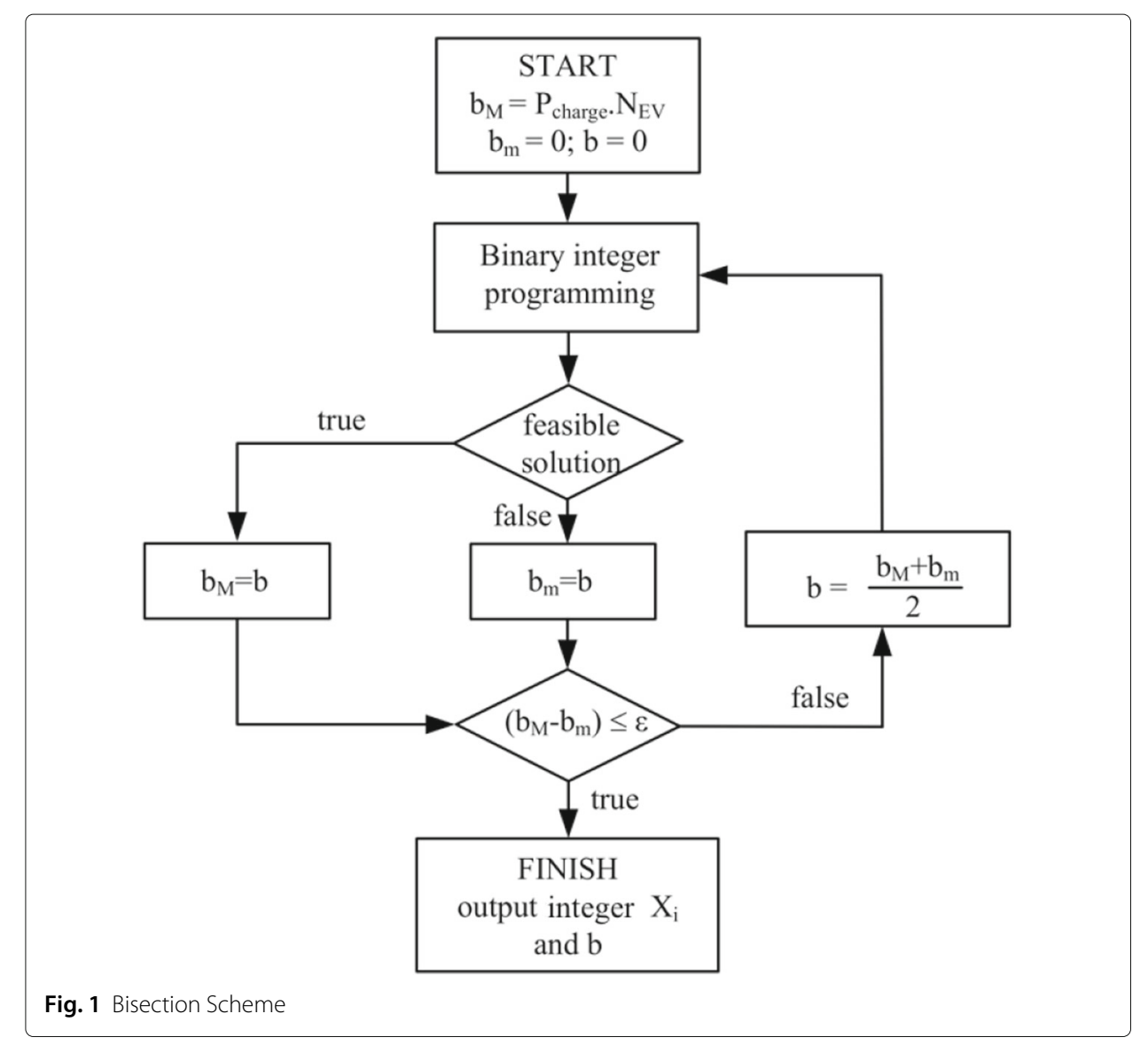

\section{Scenarios}

Baseline

As a reference scenario, we calculate the baseline peak load based on a simple first-infirst-out (FIFO) rule to charge the vehicles. Buses charge as soon as they arrive at the bus depot and remain attached to the grid until the state of charge of the battery reaches $100 \%$. The algorithm employs discrete time intervals of $10 \mathrm{~min}$.

\section{Heuristic}

To further compare results obtained, the algorithm proposed by Jahic et al. (Jahic et al. 2019) (an adaptation of the algorithm developed by Yaw et al. (Yaw and Mumey 2017)) was evaluated on the dataset. The heuristic suggested by these authors considers nonpreemptable charging jobs and a fixed charging rate. It is articulated in the following three steps:

Table 4 Vehicle Types and their Parameters, adapted from (Jahic et al. 2019)

\begin{tabular}{lll}
\hline Vehicle Type & Bus Energy Consumption $E_{\text {bus }}[\mathbf{k W h} / \mathbf{k m}]$ & Battery Size $E_{\text {bat }}[\mathbf{k W h}]$ \\
\hline SINGLE & 1.2 & 250 \\
DOUBLE & 1.6 & 300 \\
MIDI & 1.5 & 280 \\
\hline
\end{tabular}




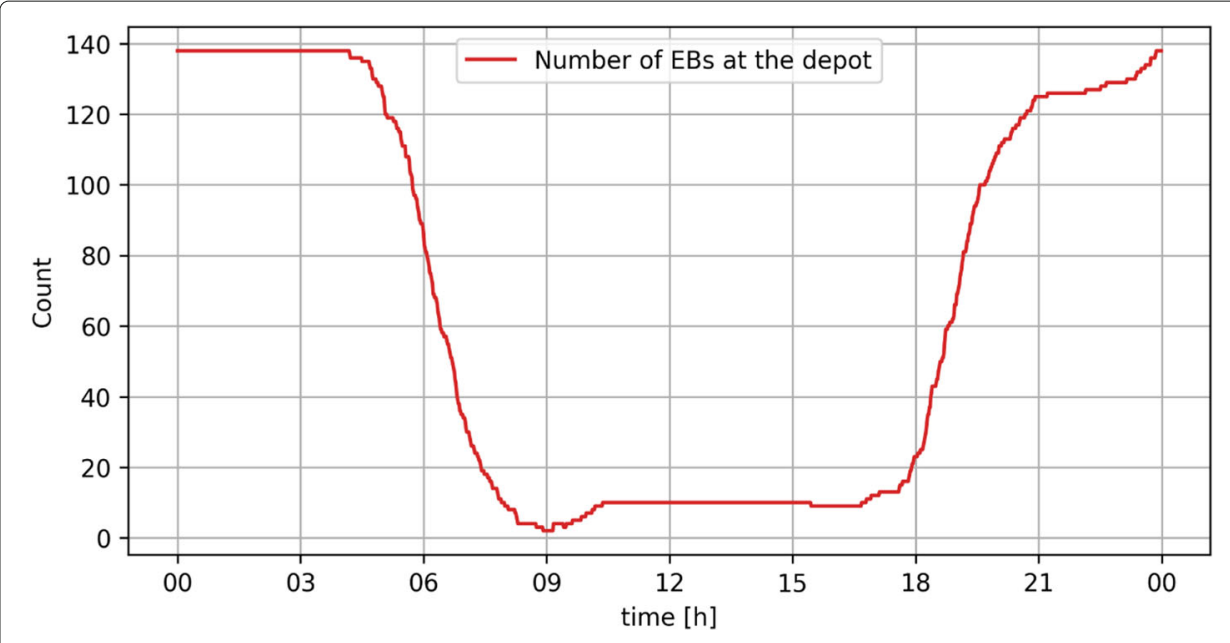

Fig. 2 Number of Buses Parked at the Depot during the Day

1 Calculate all possible charging intervals for all buses and write them into tuples defined as

$$
P_{b}=\left[s_{b}, s_{b}+l_{b}\right], \quad s_{b}=a_{b}+\delta \quad \delta=0,1,2, \ldots \delta_{b}
$$

where $a_{b}$ is the arrival time, $l_{b}$ is the number of intervals required to fully charge the vehicle, $s_{b}$ is the selected slot to start charging and $\delta_{b}$ is the shifting time of the vehicle b calculated as $\delta_{b}=d_{b}-a_{b}-l_{b}$.

2 Organize all buses ascending by their shifting time $\delta_{b}$.

3 For each task, iterate over the possible charging tuples and select the one that outputs the lowest peak in the final profile.

\section{Optimization condition scenarios}

In order to analyze the impact of varying optimization conditions on the peak load minimization and the computational time, we perform a sensitivity analysis:

Table 5 outlines the range of values different conditions take within the scenarios.

- Time Granularity (TG): The length of the discrete time intervals considered by the algorithm is varied as $60 \mathrm{~min}, 30 \mathrm{~min}$ and finally $10 \mathrm{~min}$ long. It is expected that the larger the time granularity (i.e., smaller time interval), the lower the final profile peak and the greater the computational time.

- Time-Slot Interdependence (TSI): Typical optimization analyses consider each discrete time slot to be independent of the other, allowing the scheduler to charge the

Table 5 Scenarios

\begin{tabular}{|c|c|c|c|c|}
\hline Optimization Condition Scenarios & Time Granularity & Preemption & V2G & Sub-fleet Size \\
\hline Baseline & $10 \mathrm{~min}$ & No & No & 100 \\
\hline Heuristic & $10 \mathrm{~min}$ & No & No & 100 \\
\hline Time Granularity (TG) & {$[10,30,60] \mathrm{min}$} & Yes & No & 100 \\
\hline Time-slot Interdependence (TSI) & {$[10,30,60] \mathrm{min}$} & No & No & 100 \\
\hline Bi-directional Power Flow (Bi-PF) & $10 \mathrm{~min}$ & Yes & Yes & 100 \\
\hline Fleet Splitting (FS) & $10 \mathrm{~min}$ & Yes & No & {$[10,25,50]$} \\
\hline
\end{tabular}

Bold fonts indicate the parameters varied in the corresponding scenario 
vehicle in a time-slot and interrupt charging in the next to reduce the overall peak. However, this is detrimental to the battery life. In this scenario, the time-slots are not independent of each other; the impact of disallowing the interruption of the charging procedure at each time-slot independent of the other time-slots (no preemption) on the peak reduction and computational time is assessed, without concern about the battery life.

- Bi-directional Power Flow (Bi-PF): This scenario explores the impact of enabling vehicle-to-grid (V2G) on the peak reduction and the computational time.

- Fleet Splitting (FS): The impact of splitting the entire vehicle fleet into smaller subfleets of various sizes on the peak minimization and computational time is assessed. The aim is to identify ways to speed up the computations while preserving the peak minimization effect. We assess 50 randomly chosen sub-fleets for each sub-fleet size.

The combination of optimization conditions leading to the greatest reduction in peak as well as fastest computation, as compared to the baseline, are highlighted as well. The optimizations are run on a MacBook Pro with $2.6 \mathrm{GHz}$ Intel Core i7 quad-core processor with 16 GB RAM.

\section{Results}

\section{Baseline and heuristic}

Figure 3 shows the baseline and heuristic power profiles. The daily average peak of 7,959 $\mathrm{kW}$ in the uncontrolled charging scenario of the baseline occurs in the evening between $7-8 \mathrm{PM}$, when most buses arrive back to the depot with empty batteries. The heuristic profile already leads to a considerable flattening of the power profile, with the peak reducing by $75.9 \%$ with respect to the baseline. The charging load is more evenly spread through the night, which helps flatten the evening peak.

\section{Optimization condition scenarios}

Figure 4 shows the resulting load profiles with optimized bus schedules at three time granularities, with and without preemption. The irregularity in the resulting power profiles is explained by the fixed high charging power considered in this work. Buses charge

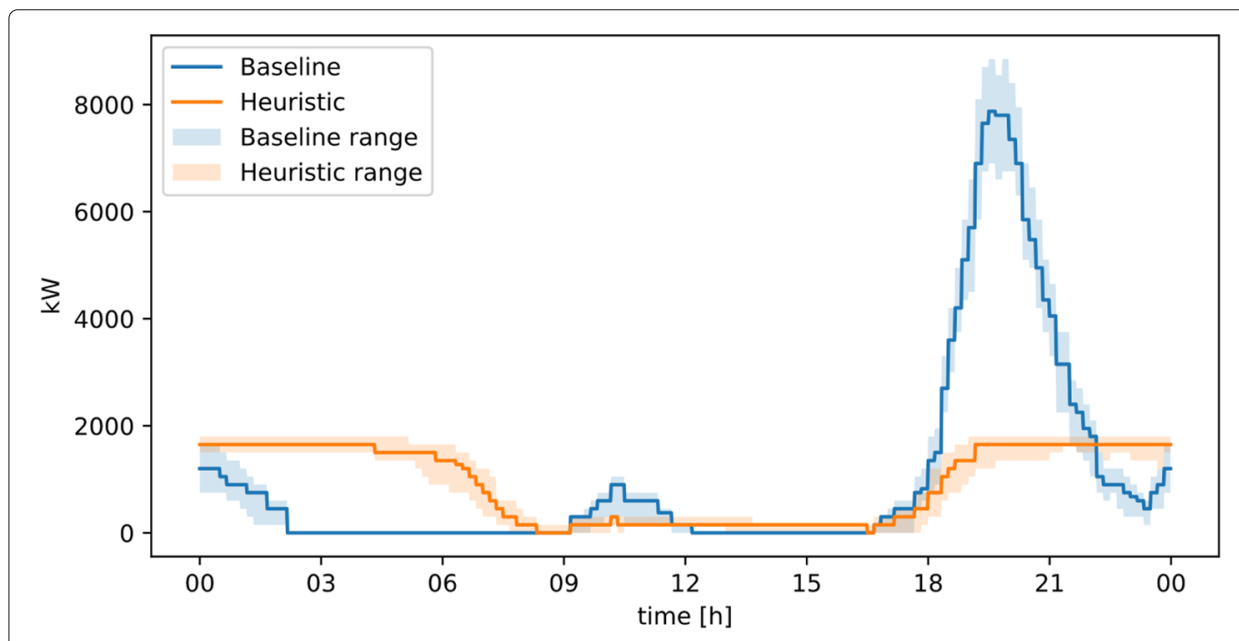

Fig. 3 Baseline and Heuristic Power Profiles 


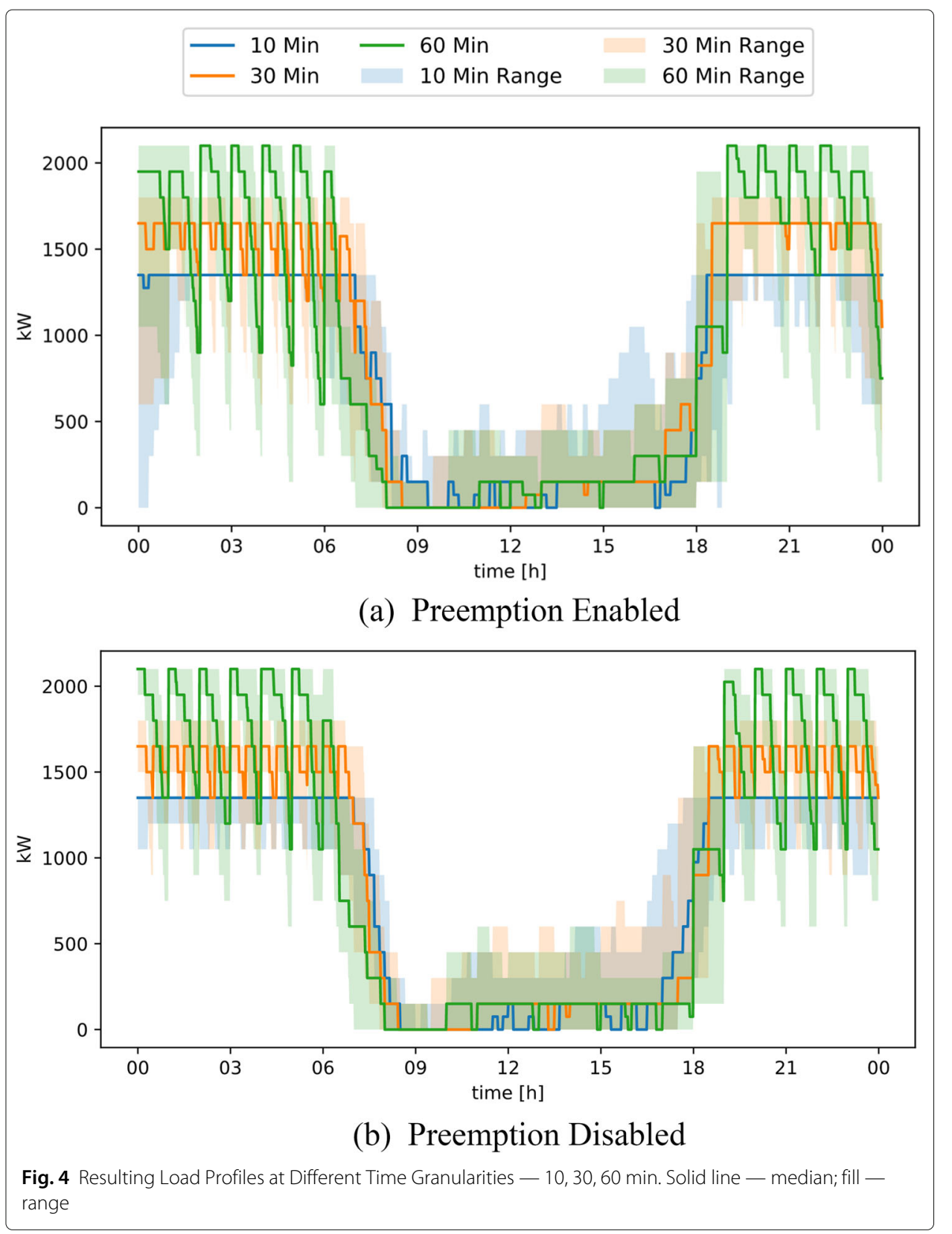

quickly and reach 100\% SOC before the current time-slot ends, thereby dropping out of the charging process and creating a trough in the power profile. In the next time-slot, the optimization schedules the same number but different buses for charging, so that the power peak remains the same. This variation reduces at large time granularities, due to the optimization being able to charge buses for a smaller time duration. The plots for the 10-min time granularity can be directly compared with the baseline and heuristic scenarios. By spacing out charging tasks equally during the night hours, our optimization algorithm results in an average peak of $1350 \mathrm{~kW}-$ a reduction of $83 \%$.

\section{Time granularity (TG)}

Figure 4a highlights the impact of the time granularity on the peak of the charging power profile of the bus depot. Shorter time-slots (large time granularity) lead to lower peaks - 
the peak with 10 -min time granularity is $1350 \mathrm{~kW}, 33 \%$ smaller than the peak for a time granularity of $60 \mathrm{~min}$. It thus becomes evident that scheduling EB charging benefits the depot sizing when the charging slots considered by the optimization are small.

\section{Time-slot interdependence (TSI)}

Figure $4 \mathrm{~b}$ shows the resulting optimal profiles without preemption. Although there are slight differences in the load profile shape as compared to the profiles when preemption is enabled (Fig. 4a), the load peaks are equal in both cases, irrespective of the time granularity. Enabling or disabling preemption has no significant impact on the peak reduction. However, the computational time severely increases when preemption is disallowed (see Table 6). For instance, the mean computation times for a time granularity of $10 \mathrm{~min}$ are $32.97 \mathrm{~s}$ and $1932.10 \mathrm{~s}$ with and without preemption, respectively -60 times more, without bringing any peak reduction, as is the case with other considered time granularities.

\section{Bi-directional power flow (Bi-PF)}

Enabling V2G leads to only $30 \%$ of optimization runs (15/50) to have a peak $11.1 \%$ lower than a bus depot without V2G. The median profiles show no difference in the peak (see Fig. 5).

\section{Fleet splitting (FS)}

Fleet splitting is one way to reduce the computational time at the expense of a higher peak. With sub-fleets, the optimization algorithm is unaware of the entire fleet. It optimizes each sub-fleet independently and aggregates the resulting schedules. Figure 6 shows how the power profiles change if the optimization is run with smaller sub-fleets in parallel. Smaller sub-fleets lead to a higher peak, as evident by the curves for sub-fleet sizes of 10 which have an aggregated average peak of $1,746 \mathrm{~kW}, 20 \%$ greater than with a sub-fleet

Table 6 Results Overview

\begin{tabular}{|c|c|c|c|c|c|c|c|}
\hline Scenario & TG (min) & Preemption & V2G & $\begin{array}{l}\text { Sub-fleet } \\
\text { (\#buses) }\end{array}$ & Size & Peak load (kW) & Computational Time (s) \\
\hline Baseline & 10 & No & No & 100 & & 7959 (390.92) & $0.001(.0004)$ \\
\hline Heuristic & 10 & No & No & 100 & & 1917 (81.82) & $0.221(.015)$ \\
\hline \multirow[t]{3}{*}{ TG } & 60 & Yes & No & 100 & & $2040(74.23)$ & $7.84(1.17)$ \\
\hline & 30 & Yes & No & 100 & & $1656(29.69)$ & $12.01(1.30)$ \\
\hline & 10 & Yes & No & 100 & & $1350(0.00)$ & $32.97(6.47)$ \\
\hline \multirow[t]{3}{*}{ TSI } & 60 & No & No & 100 & & $2040(74.23)$ & $18.57(3.80)$ \\
\hline & 30 & No & No & 100 & & $1662(41.11)$ & $64.07(10.04)$ \\
\hline & 10 & No & No & 100 & & $1350(0.00)$ & $1932.10(2710.81)$ \\
\hline $\mathrm{Bi}-\mathrm{PF}$ & 10 & Yes & Yes & 100 & & $1305(69.44)$ & $266.04(82.46)$ \\
\hline \multirow[t]{3}{*}{ FS } & 10 & Yes & No & 50 & & $1449(71.78)$ & $6.96(0.94)$ \\
\hline & 10 & Yes & No & 25 & & $1662(108.59)$ & $2.67(0.34)$ \\
\hline & 10 & Yes & No & 10 & & 1746 (138.08) & $1.06(0.13)$ \\
\hline $\begin{array}{l}\text { Lowest } \\
\text { Peak }\end{array}$ & 10 & Yes & Yes & 100 & & $1200(0.00)$ & 349.07 (45.66) \\
\hline $\begin{array}{l}\text { Shortest } \\
\text { Time }\end{array}$ & 60 & Yes & No & 100 & & 1950 & 6.01 \\
\hline $\begin{array}{l}\text { Shortest } \\
\text { Time } \\
\text { with FS }\end{array}$ & 10 & Yes & No & 10 & & 1950 & 0.812 \\
\hline
\end{tabular}

Note: Peak load values are means of 50 runs; SD indicated in parentheses 


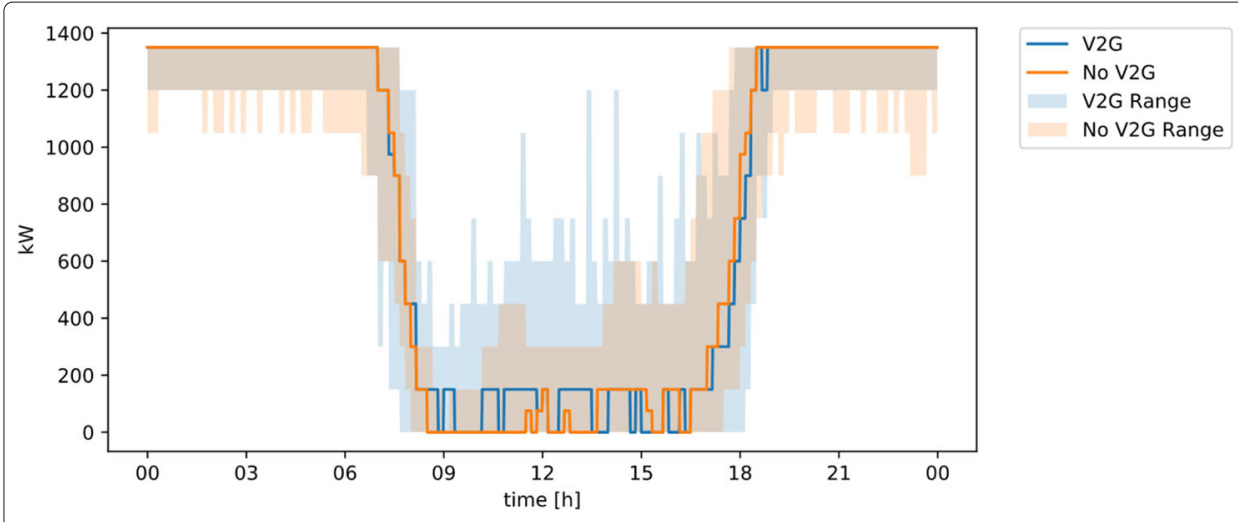

Fig. 5 Power Profile with V2G Enabled. Solid line — median; fill — range

sized 50, and 30\% greater compared to the complete fleet of 100 buses. The larger the subfleet size, the lower the peak and more homogeneous the distribution of charging tasks over the parking hours of the buses at the depot as the optimization has more information about a greater number of buses which it can consider together. On the other hand, the computational time decreases with smaller sub-fleets. A sub-fleet size of 10 buses leads to a computational time of $84 \%$ and $96 \%$ less compared with a sub-fleet sized 50 and the complete fleet, respectively.

\section{Best-case scenarios}

The bottom row of Table 6 displays the best results from individual runs. Achieving the lowest peak ( $1200 \mathrm{~kW}, 84.9 \%$ lower than the baseline scenario) is possible only with a large time granularity of $10 \mathrm{~min}, \mathrm{~V} 2 \mathrm{G}$ enabled and fleet splitting disabled, with preemption being enabled having no impact on the peak as shown before. Fast solutions need a disabling of V2G - without fleet splitting a small time granularity (60 min) is necessary to reduce computational time. With fleet splitting enabled, the time granularity can be increased to $10 \mathrm{~min}$ and still achieve the same optimal peak as without fleet splitting.

\section{Discussion}

The results highlight the importance of optimizing charging schedules, by reducing the peak by $83 \%$ on average compared to the baseline scenario and by $29.5 \%$ on average with

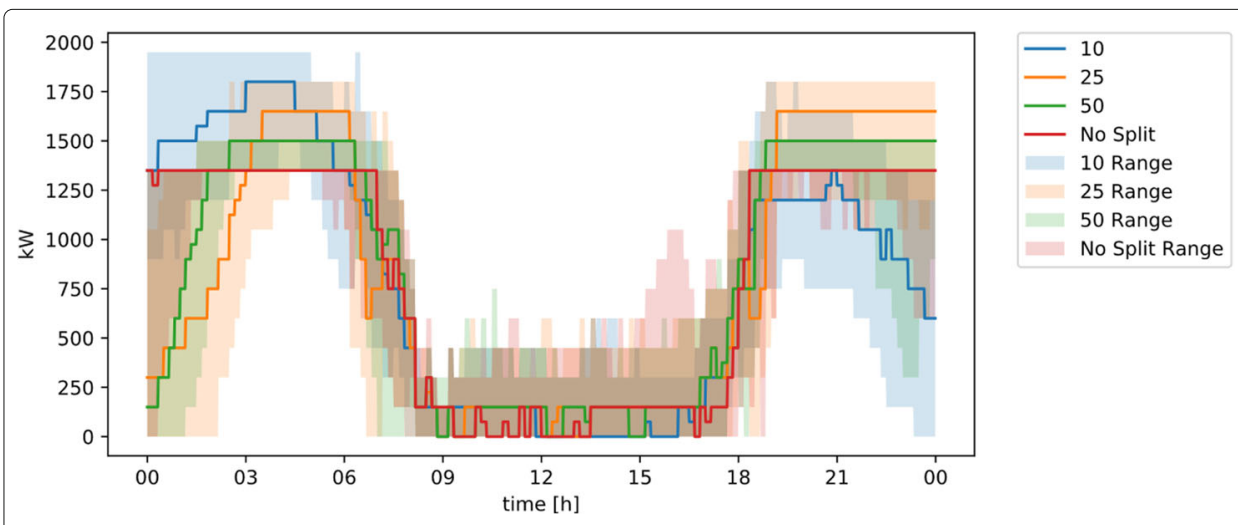

Fig. 6 Power Profiles at Different Sub-fleet Sizes. Solid line — median; fill — range 
respect to the heuristic scenario. This reduction implies a reduced need for EB charging plugs and smaller equipment sizing, leading to lower initial investment costs to realize the bus depot. It is common for industrial consumers like EB depots to procure electricity directly from electricity providers at tariffs dependent on the peak load - the optimization algorithm hence directly helps in reducing electricity costs based on such tariffs. Even though the optimization does not explicitly account for operational costs (in particular the retail cost of daily electricity needs under dynamic tariffs, for instance), the resulting load profiles are very likely to generate substantial savings in terms of operational costs. We see that, after the optimization, the total average daily electricity cost is reduced by up to $44 \%$, using simple time-of-use tariffs for electromobility needs in Zurich (EWZ). In fact, the algorithm developed shifts charging tasks to off-load periods like night, when electricity prices are typically lower.

The results also help highlight the relative importance of the time granularity of the optimization - the greater the time granularity, the lower the peak but the greater the computational time. Ideally, with discrete time intervals, a 1-min interval would provide the greatest reduction in the profile peak, although being computationally expensive. However, the resulting solution may not be practical, as having charging stations react every minute and changing the charging load abruptly can be detrimental to both the chargers and the bus batteries. A trade-off exists between EB charging slot lengths and the overall power peak, and depot planners must strive for a balance between the two.

Disabling preemption, that is, having charging slots be related to each other and thus disallowing frequent start-stops of the charging process, avoids the aforementioned problems. Since toggling preemption on/off has no impact on the peak reduction and hence the sizing of the EB depot, future research and EB depot planning can choose to always disable preemption and still achieve the same peak reduction. It leads to a more practical schedule for EB depot employees to follow as well - once a bus is plugged-in to charge, it will charge until it reaches a pre-defined level.

Empirical observations confirm the intuition that smaller sub-fleets lead to lower peak reductions, due to the limited visibility of the optimizer on the global fleet. While it does not make sense to split fleets from a pure peak reduction perspective, the computational time is much smaller for smaller sub-fleets. In time-sensitive optimizations, for instance in real-time algorithms, such fleet splitting can offer fast results in exchange for fairly modest peak increases, which could be well manageable in terms of installation and operating costs. With two small sub-fleets of 50 buses each, the peak is only $7.4 \%$ larger (corresponding to one extra charger operating in parallel), while the computational time is $81.1 \%$ lower as compared to the complete fleet. The optimization can thus be designed to achieve depot-specific planning and operational goals. However, the sub-fleet size strongly depends on the conformation of the original fleet since the aim is to maintain a similarity in terms of the distribution of the bus parking times at the depot between the sub-fleet and the global fleet.

The results also show that V2G does provide the scenario with the lowest peak load, but depot planners must be cautious. The underlying bus schedules and the variance between them are important - V2G may not help lower the peak in case of varying bus schedules. Depot planners must be wary of assuming V2G to always reduce the peak, and only invest in V2G infrastructure if such optimization results on differing bus schedules provide a definite benefit. 
Overall, the greatest peak reduction is possible by considering the entire fleet with the greatest time granularity of $10 \mathrm{~min}$ and V2G enabled. Faster results are possible by either fleet splitting or assuming the smallest time granularity of 60 mins. These 'best case' conditions, however, may not be true for different data sets. Even though the method considered is not dependent on the input data itself, computation time and peak reduction are strongly correlated to the conformation of the fleet under consideration. Nevertheless, the methodology used in the paper which involves splitting the original 138-bus dataset into random subsets of 100 buses for the analysis allows a confident assertion of the general applicability of the algorithm and the peak reduction magnitude. In any case, future research needs to test and compare the impacts of the user-set conditions considered in this work on other data sets. Limitations in the optimization algorithm also include the simplistic modelling of the EB depot, without consideration of daily operating costs, battery chemistries, weather related energy consumption, and parking space constraints. However, these limitations were acceptable, given the focus of the work to explore the impacts of user-set conditions on peak-load minimization. Future research should also consider continuous variables in the optimization algorithm, which can better replicate future EV chargers with variable power, for instance. Alternative methods to optimize the quadratic objective function, such as direct minimization instead of linearization as employed in this work, offer further opportunities for new research. Further, smart techniques for fleet splitting, such as through careful considerations of time of arrival or departure of the buses could provide a different insight into the advantages of fleet splitting for such optimization problems.

\section{Conclusion}

The modular optimization approach developed in this work takes advantage of a flexible problem formulation while maintaining the computational time reasonably low. A major difference compared to other approaches is the ability to enable preemption of the charging tasks or enable V2G and fleet splitting on demand. This helped to highlight the impact of different user-set optimization conditions on the peak load and the computational time. We find that the peak is $33 \%$ lower with greater time granularities and $11.1 \%$ lower with V2G enabled, but not in all cases. Compared to the complete fleet, a smaller sub-fleet with 10 vehicles leads to a reduction in the computational time by $96 \%$ but raises the peak by $30 \%$. These results help bus depot planners account for specific conditions, like the availability of $\mathrm{V} 2 \mathrm{G}$, in the implementation and installation of infrastructure in a new EB depot. A translation of the optimization approach used in this work to reduce operational costs is possible, but depot planners must be aware of the trade-offs between peak reduction and computational time under different user-set conditions.

\section{Acknowledgements}

The authors would like to thank Hitachi ABB Power Grids for the support to first author Enrico Toniato, and to Julia Neukam for proofreading and helping clarify key points. In addition, we thank the reviewers for comments and suggestions which helped improve and clarify this manuscript.

\section{About this supplement}

This article has been published as part of Energy Informatics Volume 4 Supplement 3, 2021: Proceedings of the 10th DACH+ Conference on Energy Informatics. The full contents of the supplement are available online at https://energyinformatics. springeropen.com/articles/supplements/volume-4-supplement-3. 


\section{Authors' contributions}

Enrico Toniato: Conceptualization, optimization algorithm design and programming, result analysis, scenario ideation, writing. Prakhar Mehta: Scenario ideation, writing, supervision. Stevan Marinkovic: Conceptualization, supervision, editing. Verena Tiefenbeck: Supervision, editing. The authors read and approved the final manuscript.

\section{Funding}

The work carried out in this study was funded by means of an internship stipend by Hitachi ABB Power Grids to Enrico Toniato. In addition, the work of researchers at the School of Business, Economics and Society at FAU was funded by the Bavarian State Ministry of Science and the Arts, in a program coordinated by the Bavarian Research Institute for Digital Transformation (bidt) of the Bavarian Academy of Sciences. Publication funding was provided by the German Federal Ministry for Economic Affairs and Energy.

\section{Availability of data and materials}

The optimization algorithm and data used in this work are available at this github repository.

\section{Declarations}

\section{Competing interests}

The authors declare that they have no competing interests.

\section{Author details}

1 Department of Mechanical and Process Engineering, ETH Zurich, Leonhardstrasse 21, LEE K, 8092 Zurich, Switzerland.

${ }^{2}$ School of Business, Economics and Society, FAU Erlangen-Nuremberg, Lange Gasse 20, 90403 Nuremberg, Germany.

${ }^{3}$ Hitachi ABB Power Grids, Bruggerstrasse 72, 5400 Baden, Switzerland. ${ }^{4}$ Department of Management, Technology and

Economics, ETH Zurich, Switzerland, Weinbergstrasse 56-58, 8092 Zurich, Switzerland.

\section{Published: 13 September 2021}

\section{References}

2050 long-term strategy — Climate Action. https://ec.europa.eu/clima/policies/strategies/2050_en. Accessed 04 May 2021

ABB (2020) Hitachi ABB Power Grids. https://www.hitachiabb-powergrids.com/ch/en. Accessed 04 May 2021

Arango Castellanos JD, Dhanasekaran Velayutha Rajan H, Rohde A-K, Denhof D, Freitag M (2019) Design and simulation of a control algorithm for peak-load shaving using vehicle to grid technology. SN Appl Sci 1(9):1-12

Buses - ACEA - European Automobile Manufacturers' Association. https://www.acea.be/automobile-industry/buses. Accessed 04 May 2021

Clairand J-M, Gonzalez M, Guerra-Terán P, Cedeño I, Escrivá G (2020) The impact of charging electric buses on the power grid. IEEE Power \& Energy Society General Meeting (PESGM). pp 1-5

Clement-Nyns K, Haesen E, Driesen J (2010) The impact of Charging plug-in hybrid electric vehicles on a residential distribution grid. IEEE Trans Power Syst 25(1):371-380

Clemente M, Fanti MP, Ukovich W (2014) Smart Management of Electric Vehicles Charging Operations: The Vehicle-to-charging Station Assignment Problem, Vol. 19

Das HS, Rahman MM, Li S, Tan CW (2020) Electric vehicles standards, charging infrastructure, and impact on grid integration: A technological review. Renew Sust Energ Rev 1(120):109618

Erdogan N, Erden F, Kisacikoglu M (2018) A fast and efficient coordinated vehicle-to-grid discharging control scheme for peak shaving in power distribution system. J Modern Power Syst Clean Energy 6(3):555-566

EWZ Electricity tariffs - Private customers - ewz. https://www.ewz.ch/de/private/strom/tarife/tarifuebersicht.html. Accessed 04 May 2021

Franco JF, Rider MJ, Romero R (2015) A Mixed-Integer Linear Programming Model for the Electric Vehicle Charging Coordination Problem in Unbalanced Electrical Distribution Systems. IEEE Trans Smart Grid 6(5):2200-2210

Gao Y, Guo S, Ren J, Zhao Z, Ehsan A, Zheng Y (2018) An electric bus power consumption model and optimization of charging scheduling concerning multi-external factors. Energies 11(8):2060

Hitachi ABB Power Grids Grid-eMotion ${ }^{\text {TM }}$ Fleet Factsheet Charging more with less. https://library.e.abb.com/ public/5c0f96dd943e499a9e395c81282a8a09/Grid_eMotion_Fleet_Factsheet_200713_jb2.pdf?x-sign= uCzk67qvbtXoXoi9ZCDIYnpannpWZTu7QRaKc+nu7O7vn5CTZChQv2EVxhs1oyyU. Accessed 26 April 2021

Houbbadi A, Bouton T, Redondo Iglesias E, Pelissier S, Trigui R (2019) A quadratic programming based optimisation to manage electric bus fleet charging. Int J Electr Hybrid Veh 11(4):289

Hu J, You S, Østergaard J, Lind M, Wu QW (2011) Optimal charging schedule of an electric vehicle fleet. In: 2011 46th International Universities' Power Engineering Conference (UPEC) VDE. pp 1-6

IEA (2020) Data and statistics, explore energy data by category, indicator, country or region. https://www.iea.org/dataand-statistics? country=WORLD\&fuel=CO2emissions\&indicator=CO2BySector. Accessed 04 May 2021

Jahic A, Eskander M, Schulz D (2019) Charging schedule for load peak minimization on large-scale electric bus depots. Appl Sci (Switzerland) 9(9):1748

Janovec M, Koháni M (2019) Exact approach to the electric bus fleet scheduling. Transp Res Procedia 40:1380-1387

Korkas CD, Baldi S, Michailidis P, Kosmatopoulos EB (2017) A cognitive stochastic approximation approach to optimal charging schedule in electric vehicle stations. In: 2017 25th Mediterranean Conference on Control and Automation (MED) IEEE. pp 484-489

Koutsopoulos I, Tassiulas L (2012) Optimal control policies for power demand scheduling in the smart grid. IEEE J Sel Areas Commun 30(6):1049-1060 
Mhaisen N, Fetais N, Massoud A (2020) Real-Time Scheduling for Electric Vehicles Charging/Discharging Using Reinforcement Learning. In: 2020 IEEE International Conference on Informatics, loT, and Enabling Technologies (ICloT). IEEE. pp 1-6

Nageshrao SP, Jacob J, Wilkins S (2017) Charging cost optimization for EV buses using neural network based energy predictor. IFAC-PapersOnLine 50(1):5947-5952

Nguyen VL, Tran-Quoc T, Bacha S, Nguyen B (2014) Charging strategies to minimize the peak load for an electric vehicle fleet. In: IECON 2014-40th Annual Conference of the IEEE Industrial Electronics Society IEEE. pp 3522-3528

Rahman S, Shrestha GB (1993) An investigation into the impact of electric vehicle load on the electric utility distribution system. IEEE Trans Power Deliv 8(2):591-597

Ramabhotla S, Bayne S, Giesselmann M (2016) Operation and Maintenance Cost Optimization in the Grid Connected Mode of Microgrid. 2016 IEEE Green Technol Conf (GreenTech) IEEE:56-61

Ranjan A, Khargonekar P, Sahni S (2014) Offline preemptive scheduling of power demands to minimize peak power in smart grids. IEEE Symp Comput Commun (ISCC) IEEE:1-6

Rotering N, llic M (2011) Optimal charge control of plug-in hybrid electric vehicles in deregulated electricity markets. IEEE Trans Power Syst 26(3):1021-1029

Škugor B, Deur J (2014) Dynamic programming-based optimization of electric vehicle fleet charging. 2014 IEEE Int Electr Veh Conf (IEVC) IEEE:1-8

Škugor B, Deur J (2015) Dynamic programming-based optimisation of charging an electric vehicle fleet system represented by an aggregate battery model. Energy 92:456-465

Sundström O, Binding C (2010) Optimization Methods to Plan the Charging of Electric Vehicle Fleets. Proc Int Conf Control Commun Power Eng:28-29

Tang W, Zhang YJ (2017) A Model Predictive Control Approach for Low-Complexity Electric Vehicle Charging Scheduling: Optimality and Scalability. IEEE Trans Power Syst 32(2):1050-1063

Turker H, Colak I (2018) Optimal Peak Shaving with Vehicle-to-Grid Capability of Electric Vehicles in Smart Grids. In: 2018 7th International Conference on Renewable Energy Research and Applications (ICRERA) IEEE. pp 1483-1488

Valckx T, Cator EA, Heres J (2019) Stochastic effects of customers on peak loads in a power distribution grid. Radboud University Nijmegen (Master's thesis)

Wang G, Xie X, Zhang F, Liu Y, Zhang D (2019) BCharge: Data-Driven Real-Time Charging Scheduling for Large-Scale Electric Bus Fleets. Proc Real-Time Syst Symp 2018-Decem:45-55

Xie S, Zhong W, Xie K, Yu R, Zhang Y (2016) Fair Energy Scheduling for Vehicle-to-Grid. IEEE Trans Neural Netw Learn Syst 27(8):1697-1707

Xu J, Wong VWS (2011) An approximate dynamic programming approach for coordinated charging control at vehicle-to-grid aggregator. In: 2011 IEEE International Conference on Smart Grid Communications (SmartGridComm) IEEE. pp 279-284

Yang S, Zhang S, Ye J (2019) A Novel Online Scheduling Algorithm and Hierarchical Protocol for Large-Scale EV Charging Coordination. IEEE Access 7:101376-101387

Yaw S, Mumey B (2017) Scheduling non-preemptible jobs to minimize peak demand. Algorithms 10(4):1-11

\section{Publisher's Note}

Springer Nature remains neutral with regard to jurisdictional claims in published maps and institutional affiliations.

\section{Submit your manuscript to a SpringerOpen ${ }^{\circ}$ journal and benefit from:}

- Convenient online submission

- Rigorous peer review

- Open access: articles freely available online

- High visibility within the field

- Retaining the copyright to your article

Submit your next manuscript at $\gg$ springeropen.com 